\title{
Expressões manuais e não manuais da Libras sob o olhar de pesquisadores da UFPR
}

\author{
Lídia da SILVA (1) \\ Universidade Federal do Paraná (UFPR)
}

\section{RESUMO}

A mesa redonda intitulada Aspectos fonético-fonológicos da Libras apresentada no evento Abralin ao Vivo contou com a participação de André Nogueira Xavier, Thiago Steven dos Santos e Elisane Conceição Alecrim. A mesa deu um panorama dos estudos que estão sendo desenvolvidos sobre articuladores manuais e não manuais no Programa de Pós Graduação em Letras da Universidade Federal do Paraná (UFPR). Em vista disso, os apresentadores abordaram tópicos de interesse da fonética-fonologia a partir de dados da Libras. Mostrou-se que, do ponto de vista articulatório, predominam expressões não manuais lexicais produzidas por mais de um ar-

OPEN ACCESS

EDITADO POR Raquel Freitag

AVALIADO POR

André Xavier

DATAS

Recebido: 27/07/2020 Aceito: $28 / 07 / 2020$ Publicado: 06/08/2020 ticulador (nas partes inferiores da face) e sem mudanças em sua configuração durante a realização do sinal. Discutiu-se, também, a respeito da variação dos recursos manuais e não manuais utilizados para expressão de intensidade em Libras e sobre a variação do parâmetro configuração de mão que persiste em diferentes modelos teóricos.

\section{ABSTRACT}

The roundtable entitled Phonetic-phonological aspects of Libras was presented at the Abralin ao Vivo by André Nogueira Xavier, Thiago Steven dos Santos and Elisane Conceição Alecrim. The panel gave an overview of the studies that are being developed on manual and non-manual articulators in the Postgraduate Program in Letters at the Federal University of Paraná (UFPR). In view of this, the speakers addressed topics of interest in phonetics-phonology based on Libras data. It was shown that, from an articulatory point of view, lexical non-manual expressions predominantly produced by 


\section{REVISTA DA ABRALIN}

more than one articulator (lower parts of the face) and without changes in their configurations during the signal articulation. It was also discussed the variation in the hand configuration parameter based on different theoretical models.

\section{PALAVRAS-CHAVE}

Expressões não manuais. Intensidade. Configuração de mão.

\section{KEYWORDS}

Non-manual expressions. Intensity. Hand configuration.

A consideração de que a comunicação em Libras ocorre apenas com base no alfabeto manual (datilológico) até pode ser presente na crença social (GESSER, 2009). Entretanto, não é para os pesquisadores, André Nogueira Xavier, Thiago Steven dos Santos e Elisane Conceição Alecrim, que compuseram a mesa redonda Aspectos fonético-fonológicos da Libras apresentada na plataforma on-line no evento Abralin Ao Vivo, no dia 24 de julho de 2020, pois estes partiram do nível linguístico fonético-fonológico para tecer suas considerações a respeito dos itens lexicais que compõem a Libras. Os três apresentadores são vinculados ao Programa de Pós-graduação em Letras da Universidade Federal do Paraná (UFPR), sendo que o primeiro é orientador dos dois últimos e todos fizeram suas falas em Libras e foram, simultaneamente, interpretados para a Língua Portuguesa oral por profissionais $^{1}$ da mesma instituição.

A respeito dessa composição destaque-se que a mesa manteve o mesmo padrão de cientificidade demonstrado nas demais que envolveram discussões sobre fonética e fonologia de línguas orais que foram apresentadas no evento, com a distinção de que nesta houve a participação de pós-graduandos. Em que pese, tal feito além de dar visibilidade para pesquisadores iniciantes, incentiva o compartilhamento do estudo em suas diferentes fases.

André Nogueira Xavier foi o primeiro a apresentar e começou dizendo que as expressões faciais e corporais (movimentos da cabeça, das diferentes partes da face, dos ombros e do torso) são tratadas em línguas de sinais como expressões não manuais (doravante ENM), as quais podem ser do tipo afetivas e/ou linguísticas/gramaticais. Ainda na introdução, demarcou as diferenças conceituais que recobrem essa categorização para os pesquisadores de línguas de sinais americana e britânica: BakerSchenk (1983), Crasborn (2006) e Pfau e Quer (2010). E, dando prosseguimento à fala assumiu Brennan (1992) como principal autor para a discussão do papel das ENM na realização de itens lexicais da Libras. Nesse ponto, esclareceu a possibilidade de as ENM demarcarem contrastes lexicais (que ocorrem quando todos os parâmetros de um sinal são iguais e a distinção da ENM acarreta em alteração de 


\section{REVISTA DA ABRALIN}

significado, como no caso de OCUPADO e NÃO-PODER), constituírem diferentes tipos de sinais (manuais, não manuais e multimodais/multicanais) e apresentarem propriedades distintas (haver ou não relação semântica entre a ENM e o sinal, serem estáveis ou dinâmicas, serem acompanhadas da oralização da palavra ou de parte dela e marcarem domínios sintáticos, se realizadas nas partes altas da face, e léxico, se realizadas nas partes baixas da face). Ademais, por meio de pesquisa realizada em 2014 com Barbosa, o palestrante notou a participação das ENM também na variação fonológica (como ocorre com o sinal de EUA que pode ser realizado com a bochecha inflada ou não).

Esse quadro teórico motivou o desenvolvimento de três pesquisas realizadas por Xavier, as quais tomaram dados extraídos de dicionários (de Capovilla e Raphael (2001) e Acessibilidade Brasil²) e que foram trazidas à mesa. Na primeira delas, em 2006, o palestrante olhou para 368 sinais (dentre os 2.274 selecionados) que apresentavam descrição das ENM e, interessado na tipologia dos sinais da Libras, constatou que a maioria predominante são sinais manuais $(83,50 \%)$, que apenas $0,20 \%$ são sinais não manuais e que $16,30 \%$ são reservados àqueles sinais considerados multicanais. O segundo estudo apresentado foi o desenvolvido em 2019, no qual, por meio da reanálise de 368 sinais multicanais, anteriormente analisados, no Software Access, Xavier pôde verificar uma baixa ocorrência de contrastividade lexical (apenas três casos), recorrência de descrições de ENM para diferentes sinais, sendo que dentre essas seis são mais frequentes, estando, entre essas, a expressão negativa na primeira posição. Constatou também que 93\% das ENM têm relação semântica com os sinais (como, por exemplo, no caso do sinal de DORMIR para o qual se usa a ENM de olhos cerrados) e que há ações bucais que coocorrem com os sinais nos casos de boca aberta, bochechas infladas e sugadas. O apresentador mencionou ainda que nesta pesquisa ele observou uma incidência maior do uso de mais de um articulador não-manual na realização de ENM lexicais em Libras e que 76\% desses articuladores são realizados na parte inferior da face. Por fim, a última pesquisa apresentada foi realizada em coautoria com Gritzenko (2019) e nesta os achados foram contrastados com os de Xavier (2019). Além da confirmação de algumas evidências, neste estudo os autores demonstraram que há ENM que são articuladas sem mudança em sua configuração e que são, portanto, estáveis (tais como para as expressões negativas e de testa franzida).

Avançando a discussão sobre análise lexical dos sinais da Libras, o mestrando Thiago Steven dos Santos tomou o turno para apresentar sua pesquisa de mestrado a qual trata sobre os recursos empregados na Libras para marcação de intensidade. Thiago adotou Wilbur, Malaia e Shay (2012) e Fuks (2016) para o seu referencial teórico. Explicou que os primeiros trabalham com intensidade na língua de sinais americana e que defendem que na realização desta, há a ocorrência de mudanças manuais (principalmente, relacionadas ao movimento do sinal) e não manuais (relacionadas à tensão, franzimento da face e inclinação da cabeça). Mencionou ainda que Fuks, que é pesquisadora da língua de sinais israelense, afirma que nesta língua a intensidade é marcada apenas por mudanças manuais. Adotou também Xavier (2014) para o qual a duplicação de mãos marca intensidade em Libras (para alguns sinais). O apresentador explicou a metodologia que usou para coleta, seleção, exclusão,

2 http://www.acessibilidadebrasil.org.br/joomla/ 


\section{REVISTA DA ABRALIN}

transcrição e tratamento dos dados de sete sinais: CHUVA, EXPERIÊNCIA, NÃO-SABER, FÁCIL, ALÍVIO, VONTADE, SOFRER. No que tange à análise qualitativa, o apresentador mencionou que seus dados se assentaram aos pressupostos de Wilbur, Malaia e Shay (2012) já que identificou articuladores não-manuais (57\% da amostra foi de sobrancelhas franzidas) e manuais (alongamento do movimento, duração da sinalização e alongamento da suspensão) presentes na realização da forma intensificada dos referidos sinais.

A última parte da mesa foi conduzida pela mestranda Elisane Conceição Alecrim que deu destaque ao parâmetro articulatório configuração de mão (doravante $\mathrm{CM}$ ) na consideração das variações fonético-fonológicas dos dados da Libras. Para tanto, Elisane lançou mão de três diferentes sistemas de notação, a saber: de Stokoe (1960), de Liddell e Johnson (1989), de Johnson e Liddell (2011 e 2012) e considerou sinais produzidos com as seguintes CM:

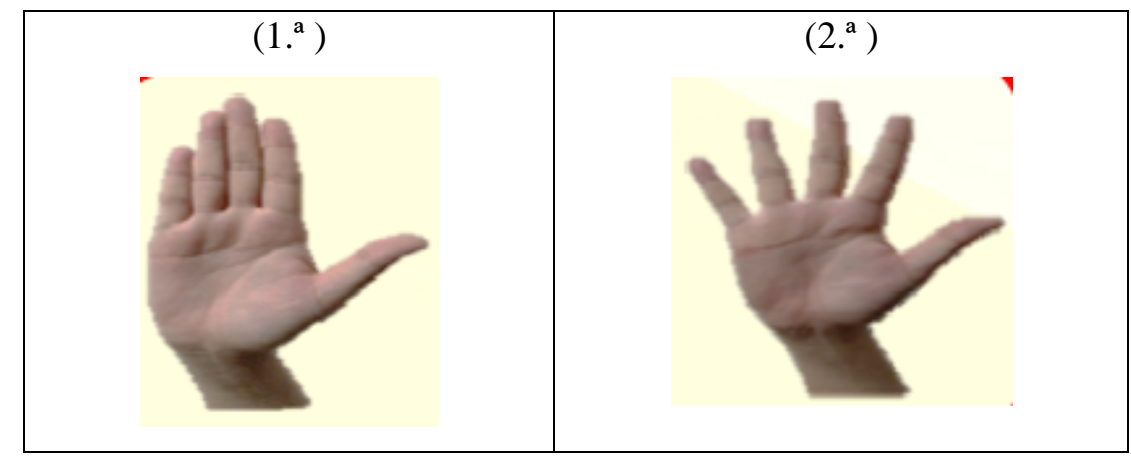

FIGURA 1 - Imagem das CM apresentadas na mesa

Fonte: slides da apresentadora

O sistema de notação de Willian Stokoe (1960), disse a apresentadora, caracteriza-se por ser de caráter fonológico e propõe 19 símbolos para as CM da língua americana de sinais e portanto, tratase de uma representação global que ignora diferenças não distintivas. A primeira imagem seria transcrita como B e a segunda como 5 nesse sistema.

O sistema de Liddell e Johnson (1989) organiza as CM em três conjuntos de traços articulatórios: configuração dos dedos, configuração do polegar e envolvimento do antebraço. A apresentadora esclareceu que segundo esse sistema as transcrições seriam Bu e $4 \mathrm{u}$ para a primeira e segunda imagem, respectivamente.

O sistema de Johnson e Liddell (2011 e 2012) é fonético e a caracterização dos articuladores é com base na anatomia da mão. Na notação, usa-se numeração para os dedos, especificação da superfície e faz-se menção aos graus de flexão dos dedos. As CM da primeira e da segunda imagem seriam transcritas, nesse sistema de notação, conforme são apresentadas a seguir: 


\section{REVISTA DA ABRALIN}
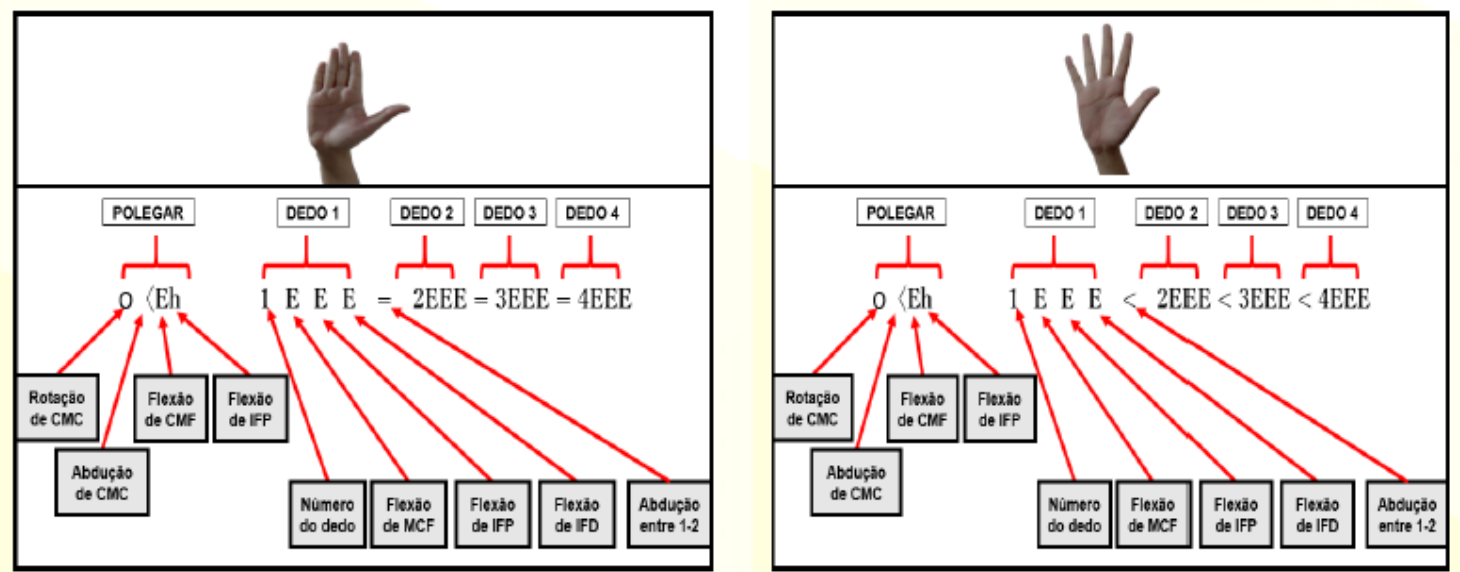

FIGURA 2 - Imagens das transcrições das CM apresentadas na Figura 1, segundo o sistema de Johnson e Liddell (2011 e 2012) Fonte: slides da apresentadora

Após mencionar a metodologia da pesquisa, a pesquisadora passou à apresentação dos resultados encontrados na análise de 26 sinais e mencionou que:

- seguindo o modelo de Stokoe (1960), a frequência de uso da CM da primeira imagem é muito maior do que da segunda, mas que todos os 12 sujeitos fizeram uso das duas variações. O sinal de LÍNGUA-DE-SINAIS foi realizado apenas com a CM da segunda imagem e o de TARDE com a da primeira;

- houve variantes identificadas em relação ao conjunto de traços articulatórios propostos no sistema de Liddell e Johnson (1989), sendo sete para a primeira imagem e dois para a segunda. Percebeu-se alta incidência de variação (seis) na realização de um mesmo sujeito. O sinal de ANDAR foi o que mais apresentou variação inter-sujeitos;

- mesmo seguindo os parâmetros do sistema de Johnson e Liddell (2011 e 2012), a notação evidenciou variação para o sinal de LÍNGUA-DE-SINAIS no que se refere à mão dominante e não dominante. O sinal de TARDE mesmo sendo monomanual apresentou variação fonética.

Abordando tais questões, essa mesa - com representatividade da UFPR - coloca-se como referência com relação aos estudos descritivos, uma vez que acrescenta conhecimento novo à área. Obviamente que, por questão de tempo, as apresentações não alcançaram a profundidade devida passando muito rapidamente em pontos importantes mas, de qualquer forma, conseguiram articular construtos de duas grandes áreas - a fonética e a fonologia - na análise de dados da Libras, assim, 


\section{REVISTA DA ABRALIN}

colaboraram com o desfazimento do mito mencionado por Gesser (2009) com relação aos itens lexicais do idioma.

Além da originalidade temática, a mesa também se destaca pela apresentação do rigor metodológico das pesquisas que conduzem e isso supera as falhas técnicas, a falta de interação com os participantes ou a falha na linearidade que, porventura, tenham havido. Pelo estilo conciso, objetivo e simples que o conteúdo foi abordado, a recomendação é que essa mesa sirva como introdução aos estudantes dos cursos de Letras Libras, de pós-graduação (Linguística das Línguas de Sinais) e profissionais em formação continuada.

\section{REFERÊNCIAS}

ASPECTOS FONÉTICOS-FONOLÓGICOS DA LIBRAS. Mesa redonda apresentada por André Nogueira Xavier, Thiago Steven dos Santos e Elisane Conceição Alecrim.[s. l., s. n.], 2020 vídeo (1h 48m 39s). Publicado pelo canal da Associação Brasileira de Linguística. Disponível em https://www.youtube.com/watch?time continue=692\&v=5aPGHLpMBcs\&feature=emb logo Acesso: 24 jul 2020.

BAKER-SHENK,C. A micro analysis of the nonmanual componentes of questions in American Sign Language.1983. Tese (Doutorado em Linguística). Universidade da Califórnia, Berkeley,1983.

BRENNAN, M. The Visual World of BSL: An Introduction. In: BRIEN, D. (Org.). Dictionary of British Sign Language/English. London: Faber \& Faber,1-134,1992.

CAPOVILLA, F. C.; RAPHAEL, W. D. Dicionário Enciclopédico Ilustrado Trilíngüe da língua de sinais brasileira. São Paulo: Edusp, 2001. 2v.

CRASBORN, O. A. Nonmanual Structures in Sign Language. In: BROWN, K. (Org.). Encyclopedia of Language E Linguistics. $2^{\mathrm{a}}$ ed., v.8. Oxford: Elsevier,668-672,2006.

FUKS, O. Intensifier actions in Israeli Sign Language. Gesture. v.2, n.15, p. 192-223. 2016.

GESSER, Audrei. Libras? que língua é essa? crenças e preconceitos em torno da língua de sinais e da realidade surda. São Paulo: Parábola Ed., 2009.

GRITZENKO, A. M.; XAVIER, A. N. Expressões não manuais lexicais em libras. In: Semana de Letras Universidade Federal do Paraná, $22^{a}, 2019$, Curitiba, PR. Anais...Curitiba:2019. p. 19-33.

JOHNSON, R. E.; LIDDELL, S. K. Toward a phonetic representation of hand configuration: The thumb. Sign Language Studies, v. 12, n.2, p. 316-333,2012.

JOHNSON, R. E.; LIDDELL, S. K. Toward a phonetic representation of hand configuration: The fingers. Sign Language Studies, v.12, n.1, p. 5-45,2011

LIDDELL, S. K.; JOHNSON, R. E. American Sign Language: The Phonological Base. In: VALLI, C.; C. LUCAS (org). Linguistic of American Sign Language: na introduction. Washington, D. C.: Clerc Books/ Gallaudet University Press, 1989.

PFAU, R; QUER, J. Nonmanuals: Their prosodic and grammatical roles. In: BRENTARI, D. (Org.). Sign languages. A Cambridge language survey. Cambridge: Cambridge University Press, 381-402,2010. 


\section{REVISTA DA ABRALIN}

STOKOE, W. Sign Language Structure: Na Outline of the Visual Communication Systems of the American Deaf". Studies in Linguistics: Occasional Papers,8, Washington, DC:GallaudetUniversityPress,1960.

XAVIER.A. N. Descrição fonético-fonológica dos sinais da língua brasileira de sinais (libras). Dissertação (Mestrado em Linguística). Departamento de Linguística, Universidade de São Paulo, São Paulo, 2006.

XAVIER, A. N.; BARBOSA, P. A. Diferentes pronúncias em uma língua não sonora? Um estudo da variação na produção de sinais da Libras, D.E.L.T.A, v.30, n.2, p. 371-413,2014.

XAVIER, A. N. Uma ou duas? Eis a questão! Um estudo do parâmetro número de mãos na produção de sinais da língua brasileira de sinais (libras). 2014. (Tese Doutorado em Linguística). Instituto de Estudos da Linguagem, Universidade Estadual de Campinas, Campinas, 2014.

XAVIER, A. N. Análise preliminar de expressões não-manuais lexicais na libras. Intercâmbio, v. 40, p. 41-66, 2019.

WILBUR, R. B., MALAIA, E. E SHAY, R. A. Degree modification and intensification in American sign language adjectives. Lecture Notes in Computer Science (Including Subseries Lecture Notes in Artificial Intelligence and Lecture Notes in Bioinformatics), 7218, p. 92-101. 2012. 